\title{
DAS RANDWERT-NORMPROBLEM FÜR EIN FASTLINEARES ELLIPTISCHES SYSTEM UND EINE ANWENDUNG*)
}

\author{
HEINRICH BEGEHR and ROBERT P. GILBERT \\ Herrn Professor R. Nevanlinna zum 80. Geburtstag gewidmet.
}

An die Ergebnisse von Bers [4] und Vekua [16] über lineare elliptische Differentialgleichungssysteme erster Ordnung, in Hilbertscher Normalform gegeben durch

$$
\begin{aligned}
& u_{x}-v_{y}=a u+b v+c \\
& u_{y}+v_{x}=\alpha u+\beta v+\gamma
\end{aligned}
$$

bzw. in komplexer Schreibweise als

$$
w_{\bar{z}}=A w+B \bar{w}+C,
$$

haben sich viele Untersuchungen und Verallgemeinerungen (vgl. [5], [6], [7], [10], [13]) angeschlossen. Die funktionentheoretischen Eigenschaften der Lösungen solcher Systeme sind bis hin zur Nevanlinnaschen Wertverteilungstheorie ([2], [4], [11], [12], [16]) entwickelt und entspringen dem auf Bers und Vekua zurückgehenden Ähnlichkeitsprinzip.

Randwertprobleme für obige Systeme werden ausführlich in [10], [16] und in [17], [18], [19], behandelt. Wie in [10] und [17] für den linearen Fall werden in [3] die Greenschen Funktionen erster und zweiter Art dazu benutzt, das RandwertNormproblem für die fastlineare Gleichung

$$
w_{\bar{z}}=f(z, w)
$$

zu lösen. Hier werden wie in [3] Existenz- und Eindeutigkeitsaussagen für dieses Problem unter einer abgeschwächten Lipschitzbedingung für die Funktion $f$ gemacht und die Ergebnisse zu einem sehr einfachen Beweis der einen (komplizierteren) Richtung des Ähnlichkeitsprinzips genutzt.

Mit anderen Methoden (Einbettungsmethode) ist (0) unter andersartigen Vor-

*) Diese Arbeit entstand, während sich der zweitgenannte Verfasser durch die Alexander von Humboldt-Stiftung mit dem „Senior U.S. Scientist Award“ ausgezeichnet im Jahre 1975 an der Freien Universität Berlin aufhielt. 
aussetzungen (zweimalige stetige Differenzierbarkeit von $f$ nach $w$ und $\bar{w}$ ) in [19] behandelt worden. Neben den verallgemeinerten analytischen Funktionen sind die approximativ analytischen Funktionen (vgl. [4], [15], [1]) Lösungen von Differentialgleichungen des Typs (0).

1. Vorbereitende Betrachtungen. Ist $\Phi$ eine konforme Abbildung des einfach zusammenhängenden Gebietes $D$ der komplexen Ebene $\mathbf{C}$ mit mehr als einem Randpunkt auf den Einheitskreis, so sind

$$
\begin{gathered}
G^{\mathrm{I}}(\zeta, z):=-\frac{1}{2 \pi} \log \left|\frac{\Phi(\zeta)-\Phi(z)}{1-\overline{\Phi(\zeta)} \Phi(z)}\right| \quad(\zeta, z \in D), \\
G^{\mathrm{II}}(\zeta, z):=-\frac{1}{2 \pi} \log |(\Phi(\zeta)-\Phi(z))(1-\overline{\Phi(\zeta)} \Phi(z))| \quad(\zeta, z \in D)
\end{gathered}
$$

die Greenschen Funktionen erster und zweiter Art für $D$. Hat $D$ einen glatten Rand $\partial \mathrm{D}$, so existiert eine Konstante $c$,

so daß

$$
c:=\frac{2}{\pi} \sup _{\zeta, z \in D}\left|\frac{\Phi^{\prime}(z)(\zeta-z)}{\Phi(\zeta)-\Phi(z)}\right| \geqq \frac{2}{\pi},
$$

$$
\left|G_{\zeta}^{k}(\zeta, z)\right| \leqq \frac{1}{2 \pi}\left|\frac{\Phi^{\prime}(\zeta)}{\Phi(\zeta)-\Phi(z)}\right| \leqq \frac{c}{4|\zeta-z|} \quad(\zeta, z \in D ; k=\mathrm{I}, \mathrm{II})
$$

Mit Hilfe dieser Funktionen läßt sich das Randwert-Normproblem

$$
w_{\bar{z}}=f(z, w),\left.\quad \operatorname{Re} w\right|_{\partial D}=\varphi, \quad \int_{\partial D} \operatorname{Im} w(\zeta)|d \Phi(\zeta)|=2 \pi C
$$

unter den Voraussetzungen

$$
\begin{gathered}
f: \hat{D} \times \mathbf{C} \rightarrow \mathbf{C}, f(\cdot, w(\cdot)) \in L_{p}(\hat{D}) \quad(w \in C(\hat{D}), \hat{D}:=D \cup \partial D) \\
\varphi \in C(\partial D), \quad C \in \mathbf{R}
\end{gathered}
$$

in die Fredholmsche Integralgleichung

$$
\begin{gathered}
w(z)=-\theta(z)+(\mathfrak{P} w)(z) \quad(z \in D), \\
\theta(z):=\int_{\partial D} \varphi(\zeta)\left[d_{n} G^{\mathrm{I}}-i d G^{\mathrm{I}}\right](\zeta, z)-i C \quad(z \in D), \\
(\mathfrak{P} w)(z):=2 \int_{D}\left\{f(\zeta, w(\zeta))\left[G_{\zeta}^{\mathrm{I}}+G_{\zeta}^{\mathrm{II}}\right](\zeta, z)+\overline{f(\zeta, w(\zeta))}\left[G_{\zeta}^{\mathrm{I}}-G_{\zeta}^{\mathrm{II}}\right](\zeta, z)\right\} d \xi d \eta \\
(\zeta=\zeta+i \eta, z \in D)
\end{gathered}
$$

umwandeln (vgl. [10], 10.4) und eine Lösung dieser Integralgleichung ist Lösung (eventuell im verallgemeinerten Sinn (vgl. [16])) des Randwert-Normproblems.

Genügt $f$ einer Bedingung der Form

$$
|f(z, w)-f(z, \omega)| \leqq(1 / c) g(z,|w-\omega|) \quad(z \in \hat{D} ; w, \omega \in \mathbf{C})
$$


mit einer geeigneten, in $\hat{D} \times[0,+\infty)$ definierten, nichtnegative Funktion $g$ und $c$ aus (1), so lassen sich aus Eigenschaften von $g$ Existenz- und Eindeutigkeitsaussagen für die Lösung von (3) herleiten.

2. Existenz einer Lösung. Die Funktion $g$ in (4) sei in der zweiten Veränderlichen stetig und in $x=0$ gleichgradig bezüglich $z \in \hat{D}$ linksseitig stetig, $g(z, 0) \equiv 0$ in $\hat{D}$ und es existiere eine positive Konstante $k$, so da $\beta$

$$
g(z, x) \leqq g(z, k) \quad(z \in \hat{D}, 0 \leqq x \leqq k)
$$

und

$$
|\theta(z)|+\int_{D}\{c|f(\zeta, 0)|+g(\zeta, k)\} \frac{d \xi d \eta}{|\zeta-z|} \leqq k \quad(z \in D) .
$$

Da dann $-\theta+\mathfrak{B}$ eine stetige Abbildung der kompakten, konvexen Menge

$$
A:=\left\{w: w \in C(\hat{D}), \operatorname{Max}_{z \in \bar{D}}|w(z)| \leqq k,\left.\operatorname{Re} w\right|_{\partial D}=\varphi, \int_{\partial D} \operatorname{Im} w(\zeta)|d \Phi(\zeta)|=2 \pi C\right\}
$$

des Banachraumes $C(\hat{D})$ in sich darstellt, gibt es nach dem Schauderschen Fixpunktsatz in $A$ eine Lösung von (3).

3. Eindeutigkeit der Lösung. Die Eindeutigkeit der Lösung in $A$ kann unter folgenden Voraussetzungen gesichert werden:

$$
\begin{gathered}
g(z, 0) \equiv 0, \quad g(z, x) \leqq g(z, y) \quad(z \in \hat{D}, 0 \leqq x \leqq y) . \\
\int_{D} g(\zeta, 2 k) \frac{d \xi d \eta}{|\zeta-z|} \leqq k \quad(z \in \hat{D}) .
\end{gathered}
$$

(9) Der auf der Menge der in $\hat{D}$ stetigen, reellen Funktionen durch

gegebene Operator $\mathfrak{\Im}$ hat auf

$$
(\mathfrak{I} x)(z):=\int_{D} g(\zeta, x(\zeta)) \frac{d \xi d \eta}{|\zeta-z|} \quad(z \in \hat{D})
$$

$$
C_{k}:=\{x: x \in C(\hat{D}), 0 \leqq x(z) \leqq k(z \in \hat{D})\}
$$

nur den Fixpunkt $x=0$.

Sind nämlich $w$ und $\omega$ zwei Lösungen der Integralgleichung (3) in $A$, so folgt mit (4)

$$
|w-\omega| \leqq \mathfrak{J}|w-\omega| .
$$

Wegen (7) bis (9) hat diese Ungleichung nur die Lösung $|\mathfrak{w}-\omega|=0$. Eine Funktion $g$, die den unter 2. und 3. gestellten Forderungen genügt und die die Eindeutigkeit der Lösung in ganz $C(\hat{D})$ ergibt, ist (Lipschitzbedingung!)

$$
\begin{gathered}
g(z, x):=g_{0}(z) x, \quad 0 \leqq g_{0}(z) \quad(z \in \hat{D}), \quad g_{0} \in L_{p}(\hat{D}) \quad(2<p), \\
\int_{D} g_{0}(\zeta) \frac{d \xi d \eta}{|\zeta-z|}<1 \quad(z \in \hat{D}) .
\end{gathered}
$$


Gilt außerdem noch $f(z, 0) \equiv 0$ in $\hat{D}$, so ist mit den obigen Aussagen das RandwertNormproblem für approximativ analytische Funktionen gelöst.

Die in (6), (8), (10) enthaltenden Integralbedingungen sind bei gegebener Funktion $f$ für genügend kleine Gebiete $D$ erfüllbar, so daß die Ergebnisse lokale Aussagen darstellen.

4. Anwendung. Die vorangegangene Existenzaussage läßt sich zu einem neuen Beweis des zweiten Teils des folgenden auf $L$. Bers [4] und I. N. Vekua [16] zurückgehenden Ähnlichkeitsprinzips nutzen:

(i) Jede Lösung der Differentialgleichung

$$
w_{\bar{z}}=a(z) w+b(z) \bar{w} \quad\left(a, b \in L_{p}(D), 2<p\right)
$$

kann mit Hilfe einer geeigneten analytischen Funktion $f$ durch

$$
w=f e^{\omega}
$$

dargestellt werden, wobei $\omega$ eine beschränkte und Hölder-stetige Funktion ist, die etwa durch

$$
\begin{gathered}
\omega(z):=\int_{D}\left\{\Omega(\zeta)\left[G_{\zeta}^{\mathrm{I}}+G_{\zeta}^{\mathrm{II}}\right](\zeta, z)+\overline{\Omega(\zeta)}\left[\dot{G}_{\zeta}^{\mathrm{I}}-G_{\zeta}^{\mathrm{II}}\right](\zeta, z)\right\} d \zeta d \eta, \\
\Omega(z):=a(z)+b(z) \frac{\overline{w(z)}}{w(z)} \quad(z \in D)
\end{gathered}
$$

gegeben werden kann. Hieraus folgt

$$
|w(z)|=|f(z)| \quad(z \in \partial D) .
$$

(ii) $\mathrm{Zu}$ jeder in $D$ analytischen Funktion $f$ kann eine Lösung von (11) gefunden werden, die zu $f$ in einer Beziehung der Form (12) steht.

Während der erste Teil dieses Prinzips einfach zu beweisen ist, bereitet der zweite Teil einige Mühe (vgl. [3], § 17 und [10], 11.5). Verlangt man, daß zu vorgegebener, in $D$ analytischer Funktion $f$ eine Lösung von (11) in der Form (12) existiert, so muß $\omega$ der nichtlinearen Differentialgleichung

$$
\omega_{\bar{z}}=F(z, \omega), \quad F(z, \omega):=a(z)+b(z) \frac{\overline{f(z)}}{f(z)} \exp [-2 i \operatorname{Im} \omega]
$$

genügen. Es gelten

$$
\begin{gathered}
|F(z, \omega)| \leqq|a(z)|+|b(z)| \quad(z \in D, \omega \in \mathbf{C}), \\
|F(z, \omega)-F(z, \tilde{\omega})|=2|b(z)||\sin \operatorname{Im}(\omega-\tilde{\omega})| \quad(z \in D ; \omega, \tilde{\omega} \in \mathbf{C}) .
\end{gathered}
$$

Für (13) ist das Randwert-Normproblem nach 2. in $A$ lösbar, wenn $k>0$ so gewählt wird, daß

$$
|\theta(z)|+c \int_{D}(|a(\zeta)|+|b(\zeta)|) \frac{d \xi d \eta}{|\zeta-z|} \leqq k \quad(z \in D) .
$$


Die Eindeutigkeit der Lösung kann unter der Bedingung

$$
2 c \int_{D}|b(\zeta)| \frac{d \xi d \eta}{|\zeta-z|}<1
$$

gesichert werden. Da in diesem Fall $\mathfrak{P}$ ein kontrahierender Operator ist, kann der Banachsche Fixpunktsatz angewandt und die Lösung iterativ bestimmt werden.

Für den zweiten Teil des Ähnlichkeitsprinzips reicht der Existenznachweis. Beschränkt man sich noch auf die homogene Randvorgabe

$$
\left.\operatorname{Re} \omega\right|_{\partial D}=0,
$$

so gilt für die Lösung $w=f \exp \omega$ von (11)

$$
|w(z)|=|f(z)| \quad(z \in \partial D),
$$

eine Bedingung wie sie auch im ersten Teil des Ähnlichkeitsprinzips angegeben wurde.

\section{Litera tur}

[1] Begehr, H.: Zur Wertverteilung approximativ analytischer Funktionen. - Arch. Math. (Basel) 23, 1972, 41-49.

[2] Begehr, H.: Die logarithmische Methode in der Wertverteilungstheorie pseudoanalitytischer Funktionen. - Ann. Acad. Sci. Fenn. Ser. A I 549, 1973, 1 - 17.

[3] Begehr, H., und R. P. Gilbert: Über das Randwert-Normproblem für ein nichtlineares elliptisches System. - Function Theoretic Methods for Partial Differential Equations, Darmstadt, 1976. Lecture Notes in Mathematics 561, Springer Verlag, BerlinHeidelberg-New York, 1976, 112-122.

[4] Bers, L.: Theory of pseudo-analytic functions. - Vorlesungsausarbeitung, New York University, 1953.

[5] BoJARSKI, В. V.(Б. В. БОЯРСКИЙ): Теория обобщенного анапитического вектора. - Ann. Polon. Math. 17, 1966, 281-320.

[6] Gilbert, R. P.: Constructive methods for elliptic partial differential equations. - Lecture Notes in Mathematics 365, Springer-Verlag, Berlin-Heidelberg-New York, 1974.

[7] Gilbert, R. P., and G. Hile: Generalized hyperanalytic function theory. - Trans. Amer. Math. Soc. 195, 1974, 1-29.

[8] Gilbert, R. P., and G. C. Hsiao: On Dirichlet's problem for quasilinear elliptic equations. Lecture Notes in Mathematics 430, Springer-Verlag, Berlin-Heidelberg-New York, 1974, 184-236.

[9] Gilbert, R. P., and R. J. Weinacht: Iterative schemes for elliptic systems. - Ibid. 253-260.

[10] HaACK, W., und W. Wendland: Vorlesungen über partielle und Pfaffsche Differentialgleichungen. - Birkhäuser Verlag, Basel-Stuttgart, 1969.

[11] Habetha, K.: Über die Werteverteilung pseudoanalytischer Funktionen. - Ann. Acad. Sci. Fenn: Ser A I 406, 1967, 1-20.

[12] Habetha, K.: On zeros of elliptic systems of first order in the plane. - Function theoretic methods in differential equations. Pitman Publ. London-San Francisco-Melbourne, 1976, 45-62.

[13] TutschKe, W.: Konstruktion von globalen Lösungen mit vorgeschriebenen Singularitäten bei partiellen komplexen Differentialgleichungssystemen. - S.-B. Sächs. Akad. Wiss. Leipzig Math.-Natur. Kl. 109, Heft 7, 1972. 
[14] Tutschke, W.: Theorie und Anwendungen morpher Funktionen. - Beiträge Anal. 4, 1972 , $167-175$.

[15] Tutschke, W.: Ein Differenzierbarkeitssatz für approximativ analytische Funktionen. - Math. Z. $142,1975,27-31$.

[16] Vekua, I. N.: Verallgemeinerte analytische Funktionen. - Akademie Verlag, Berlin, 1963.

[17] Wendland, W.: Über Ähnlichkeitsprinzip und Randwertaufgaben für verallgemeinerte analytische Funktionen. - Applicable Anal. 2, 1972, 101-110.

[18] Wendland, W.: On boundary value problems of generalized analytic functions. - Conf. Theory ordinary partial diff. equ., Dundee/Scottland 1972. Lecture Notes in Mathematics 280, Springer-Verlag, Berlin-Heidelberg-New York, 1972, 190-201.

[19] Wendland, W. L.: An integral equation method for generalized analytic functions. - Lecture Notes in Mathematics 430, Springer-Verlag, Berlin-Heidelberg-New York, 1974, $414-452$.

Freie Universität Berlin

I. Math. Institut

Hüttenweg 9

D 1 Berlin 33

Eingegangen am 23. Juli 1976
University of Delaware

Department of Mathematics

Newark, Delaware 19711

USA 